\title{
Blackbody Radiation Shifts and Theoretical Contributions to Atomic Clock Research
}

\author{
M. S. Safronova ${ }^{1}$, Dansha Jiang, Bindiya Arora, Charles W. Clark, M. G. Kozlov, \\ U. I.Safronova, and W. R. Johnson
}

\begin{abstract}
A review of theoretical calculations of black-body radiation (BBR) shifts in various systems of interest to atomic clock research in presented. Calculations for monovalent systems, such as $\mathrm{Ca}^{+}, \mathrm{Sr}^{+}$, and $\mathrm{Rb}$ are carried out using a relativistic all-order single-double method, where all single and double excitations of the Dirac-Fock wave function are included to all orders of perturbation theory. A recently developed method for accurate calculations of BBR shifts in divalent atoms such as $\mathrm{Sr}$ is discussed. This approach combines the relativistic all-order method and the configuration interaction method. The evaluation of uncertainties in theoretical values of BBR shifts is discussed in detail.
\end{abstract}

\section{INTRODUCTION}

The current definition of a second in the International System of Units (SI) is based on the microwave transition between the two hyperfine levels $\left(F=4\right.$ and $F=3$ ) of the ${ }^{133} \mathrm{Cs}$ ground state. The present relative standard uncertainty of the Cs microwave frequency standard is approximately $4 \times 10^{-16}$ [1]. The operation of atomic clocks is generally carried out at room temperature, whereas the definition of the second refers to the clock transition in an atom at absolute zero. This implies that the clock transition frequency should be corrected for effects of finite temperature, of which the leading contributor is the blackbody radiation (BBR) shift. The BBR shift at room temperature effecting the $\mathrm{Cs}$ microwave frequency standard has been calculated to high accuracy $(0.35 \%)$ in Refs. [2], [3] implying a $6 \times 10^{-17}$ fractional uncertainty. These calculations are in agreement with a $0.2 \%$ measurement [4].

A significant further improvement in frequency standards is possible with the use of optical transitions, since frequencies of feasible optical clock transitions are five orders of magnitude larger than the relevant microwave transition frequencies. Significant recent progress in optical spectroscopy and measurement techniques has led to the achievement of relative standard uncertainties in optical frequency standards that are comparable to the Cs microwave benchmark. In 2006, the International Committee for Weights and Measures (CIPM) recommended that the following transitions frequencies be used as secondary representations of the second [5]: ground-state hyperfine microwave transition in ${ }^{87} \mathrm{Rb}$ [6], [7], $5 s^{2} S_{1 / 2}-4 d^{2} D_{5 / 2}$ optical transition of the ${ }^{88} \mathrm{Sr}^{+}$ion [8], [9], $5 d^{10} 6 s^{2} S_{1 / 2}(F=0)-5 d^{9} 6 s^{2}{ }^{2} D_{5 / 2}(F=2)$ optical transition in ${ }^{199} \mathrm{Hg}^{+}$ion [10], [11], $6 s^{2} S_{1 / 2}(F=0)-$ $5 d^{2} D_{5 / 2}(F=2)$ optical transition in ${ }^{171} \mathrm{Yb}^{+}$ion [12], [13] and $5 s^{2}{ }^{1} S_{0}-5 s 5 p^{3} P_{0}$ transition in ${ }^{87} \mathrm{Sr}$ neutral atom [14],

\footnotetext{
${ }^{1}$ Email: msafrono@udel.edu
}

[15], [16]. With better stability and accuracy, as well as extremely low systematic perturbations, such optical frequency standards can reach a systematic fractional uncertainty of order $10^{-18}$ [9], [17]. The ability to develop more precise optical frequency standards will open ways of improving global positioning systems, tracking deep-space probes, performing accurate measurements of fundamental constants, and testing underlying postulates of physics.

The major contributions to systematic frequency shifts come from Stark shifts; the BBR shift being one of the most important contributions at room temperature for many of the frequency standards. Experimental measurements of the BBR shifts are difficult. In this paper, we review the current status of the theoretical calculations of BBR shifts in various systems of interest to the development of both microwave and optical frequency standards. New preliminary result for the BBR shift of the ground-state hyperfine microwave transition in ${ }^{87} \mathrm{Rb}$ is presented [18]. The evaluation of BBR shifts and their uncertainties in optical frequency standards in monovalent ions, such as $\mathrm{Ca}^{+}$[19] and $\mathrm{Sr}^{+}$[20] is discussed. New method for accurate calculations of the BBR shifts in divalent atoms such as $\mathrm{Sr}$ is outlined. This approach combines the relativistic all-order method and the configuration interaction (CI) method [21]. This method is generally applicable, i.e. not restricted to the specific type of the system.

The paper is organized as follows. We start by providing a brief general introduction into the calculation of the BBR shifts in Section II. BBR shifts of the ground-state hyperfine microwave transitions are discussed in Section III. BBR shifts of optical frequency standards with monovalent ions and their uncertainties are discussed in Section IV. BBR shifts of optical frequency standards with divalent ions and the recent development of a CI + all-order approach are discussed in Section V. A summary of results for fractional uncertainties $\delta \nu / \nu_{0}$ due to BBR shift and the fractional error in the absolute transition frequency induced by the BBR shift uncertainty in various frequency standards in given in SectionVI.

\section{THEORETICAL CALCULATION OF BBR SHIFTS}

The electrical field $E$ radiated by a blackbody at temperature $T$, as given by Planck's law,

$$
E^{2}(\omega) d \omega=\frac{8 \alpha^{3}}{\pi} \frac{\omega^{3} d \omega}{\exp \left(\omega / k_{B} T\right)-1},
$$

induces a nonresonant perturbation of atomic transitions at room temperature [22], [23]. The average electric field radiated 
by a blackbody at temperature $T$ is

$$
\left\langle E^{2}\right\rangle=(831.9 \mathrm{~V} / \mathrm{m})^{2}\left(\frac{T(K)}{300}\right)^{4} \text {. }
$$

Assuming that the system evolves adiabatically, the frequency shift of an atomic state due to such an electrical field can be related to the static electric-dipole polarizability $\alpha_{0}$ by (see Ref. [24])

$$
\delta \nu=-\frac{1}{2}(831.9 \mathrm{~V} / \mathrm{m})^{2}\left(\frac{T(K)}{300}\right)^{4} \alpha_{0}(1+\eta),
$$

where $\eta$ is a small dynamic correction due to the frequency distribution and only the electric-dipole transition part of the contribution is considered. Contributions from M1 and E2 transitions are suppressed by factors of $\alpha^{2}$ [24]. The overall BBR shift of the clock frequency is the difference between BBR shifts of the initial and final levels involved in the transition. Therefore, theoretical evaluation of the BBR shift requires accurate calculation of static scalar polarizabilities of the relevant states. The effect of the tensor part of the polarizability in the cases where it is non-zero averages out due to the isotropic nature of the electric field radiated by the blackbody.

In the case of the optical transitions, the lowest (second) order polarizabilities of the clock states are different. In the case of the ground-state hyperfine microwave frequency standards, the lowest (second) order polarizabilities of the clock states are identical and the lowest-order BBR shift vanishes. To evaluate the BBR shift, third-order $F$-dependent polarizabilities must be calculated. Therefore, we treat these cases separately. We note that from the theoretical standpoint, the number of the valence electrons (and the presence of core holes) defines the type (and final accuracy) of the approach. For example, the calculations of the BBR shift in $\mathrm{Ca}^{+}$and $\mathrm{Sr}^{+}$are effectively the same, but the treatment of $\mathrm{Sr}^{+}$and $\mathrm{Sr}$ are completely different. The main sources of uncertainties in these cases are also different for different systems and are discussed separately for each distinct case.

\section{MICROWAVE FREQUENCY STANDARDS}

The third-order $F$-dependent static polarizability $\alpha_{F}^{(3)}(0)$ required for the evaluation of the BBR shift for the microwave frequency standards in $\mathrm{Rb}$ and $\mathrm{Cs}$ can be evaluated as [24]

$$
\alpha_{F}^{(3)}(0)=A g_{I} \mu_{n}(2 T+C+R),
$$

where $A$ is an angular coefficient, $g_{I}$ is the nuclear gyromagnetic ratio, and $\mu_{n}$ is nuclear magneton. The quantities $T, C$, and $R$ contain terms with two electric-dipole reduced matrix elements $\langle i\|D\| j\rangle$ and one matrix element of the magnetic hyperfine operator $\left\langle i\left\|\mathcal{T}^{(1)}\right\| j\right\rangle$. For example, term $T$ is given by [24]

$$
T=\sum_{m \neq v} \sum_{n \neq v} A_{1} \delta_{j_{n} j_{v}} \frac{\langle v\|D\| m\rangle\langle m\|D\| n\rangle\left\langle n\left\|\mathcal{T}^{(1)}\right\| v\right\rangle}{\left(E_{m}-E_{v}\right)\left(E_{n}-E_{v}\right)}
$$

where $A_{1}$ is an angular coefficient and sums over $m, n$ run over all possible states allowed by the selection rules. The sums are made finite with the use of finite B-spline basis set in a spherical cavity. Two other terms, $C$ and $R$, contain sums of the $D \mathcal{T}^{(1)} D$ and $\mathcal{T}^{(1)} D^{2}$ terms. It is practical to separate sum over states to the main contribution calculated using the high-precision all-order (or experimental) matrix elements and experimental energies, and the remainder ("tail") calculated using either Dirac-Fock or random-phase (RPA) approximation. In Cs calculation [24], the main term contained sum over orbitals with the principal quantum number $n \leq 12$. While tail contribution is small, it is significant (on the order of $7 \%$ in Cs [24]). Off-diagonal hyperfine matrix elements between the $s$-states $\left\langle n s\left\|\mathcal{T}^{(1)}\right\| n^{\prime} s\right\rangle$ can be also evaluated using the formula

$$
\left|\left\langle n s\left\|\mathcal{T}^{(1)}\right\| n^{\prime} s\right\rangle\right|=\sqrt{\left\langle n s\left\|\mathcal{T}^{(1)}\right\| n s\right\rangle\left\langle n^{\prime} s\left\|\mathcal{T}^{(1)}\right\| n^{\prime} s\right\rangle}
$$

that is useful for the cases where accurate values of the hyperfine constants $A$ are available. Therefore, the calculation of the BBR shift reduces to the evaluation of the electric-dipole and magnetic hyperfine matrix elements. Accurate evaluation of these quantities, in particular for heavy systems, requires allorder approaches where dominant electronic correlation terms are summed to all orders of many-body perturbation theory. Two such approaches implemented in significantly different ways have been used for the calculation of the blackbody radiation shifts: the relativistic all-order method (or linearized coupled-cluster method LCCSD[pT] method) [3], [18], [25], [26], [27] and perturbation theory in the screened Coulomb interaction (PTSCI) [2], [28] (also referred to as the correlation potential method). We describe the LCCSD[pT] method briefly below and summarize PTSCI method in Section III-C.

The relativistic all-order method including single, double, and partial valence triple excitations (or LCCSDpT) was applied to accurate calculations of energies, transition amplitudes, hyperfine constants, static and dynamic electricdipole polarizabilities, quadrupole and octupole polarizabilities, magic wavelengths, atomic quadrupole moments, $C_{3}$ and $C_{6}$ coefficients, isotope shifts and other properties of monovalent atoms (Li, Na, Mg II, Al III, Si IV, P V, S VI, $\mathrm{K}$, Ca II, In, In-like ions, Ga, Ga-like ions, Rb, Cs, Ba II, Tl, Fr, Ra II, Th IV, other Fr-like ions, Ra II ) as well as the calculation of parity-violating amplitudes in $\mathrm{Cs}$, Fr, and $\mathrm{Ra}^{+}$. We refer the reader to review [29] and references therein for a detailed description of this method, its extensions, and applications. The relativistic all-order method is applicable to the calculation of the monovalent systems, i.e. alkali-metal atoms, $\mathrm{Ca}^{+}, \mathrm{Sr}^{+}, \mathrm{Zn}^{+}$, etc. For example, it can be used to evaluate the properties of the ground or $5 d^{10} 6 p$ excited state of $\mathrm{Hg}^{+}$, but not the properties involving $5 d^{9} 6 s^{2}$ configuration in $\mathrm{Hg}^{+}$since it is not a single valence electron state (but twoparticle and one-hole state). We discuss an all-order approach capable of treating more complicated systems in Section V.

\section{A. The all-order LCCSD[pT] method}

The point of departure for our calculation is the relativistic no-pair Hamiltonian $H=H_{0}+V_{I}$ [30] expressed in second 
quantization as

$$
\begin{aligned}
H_{0} & =\sum_{i} \epsilon_{i}: a_{i}^{\dagger} a_{i}:, \\
V_{I} & =\frac{1}{2} \sum_{i j k l} v_{i j k l}: a_{i}^{\dagger} a_{j}^{\dagger} a_{l} a_{k}:,
\end{aligned}
$$

in the case of frozen-core $V^{N-1}$ Dirac-Fock potential. The quantities $v_{i j k l}$ are two-particle matrix elements of the Coulomb interaction $g_{i j k l}$ or Coulomb + Breit interaction $g_{i j k l}+b_{i j k l}, \epsilon_{i}$ in Eq. (7) is the eigenvalue of the Dirac equation, $a_{i}^{\dagger}, a_{i}$ are creation and annihilation operators, and : : designated normal ordering of operators with respect to the core. In the linearized LCCSDpT approach, the atomic wave function of a monovalent atom in a state $v$ is given by an expansion

$$
\begin{aligned}
\left|\Psi_{v}\right\rangle=[1+ & \sum_{m a} \rho_{m a} a_{m}^{\dagger} a_{a}+\frac{1}{2} \sum_{m n a b} \rho_{m n a b} a_{m}^{\dagger} a_{n}^{\dagger} a_{b} a_{a}+ \\
& +\sum_{m \neq v} \rho_{m v} a_{m}^{\dagger} a_{v}+\sum_{m n a} \rho_{m n v a} a_{m}^{\dagger} a_{n}^{\dagger} a_{a} a_{v} \\
& \left.+\frac{1}{6} \sum_{m n r a b} \rho_{m n r v a b} a_{m}^{\dagger} a_{n}^{\dagger} a_{r}^{\dagger} a_{b} a_{a} a_{v}\right] a_{v}^{\dagger}\left|\Psi_{C}\right\rangle,
\end{aligned}
$$

9) $a b$ initio LCCSD or LCCSDpT values are used in place of the experimental data and the final results are compared [3]. The uncertainty of the experimental energy values [34], [35] is negligible. Where the experimental (electric-dipole or hyperfine) matrix elements were used, the experimental uncertainties were taken. Uncertainty in theoretical matrix elements are assigned based on a comparison of theoretical and experimental values, estimation of the size of the correlation corrections, and the importance of the higher-order terms for particular matrix elements. An estimate of the uncertainty in the tail contribution can be carried out in the same fashion (by assigning the uncertainties to the individual terms) or by estimating the accuracy of the DHF approach for the entire tail. This issue will be addressed in detail in Section IV where the tail contribution is particularly large for polarizabilities of $n d$ states. The accuracy of the tail contribution was also verified by replacing lowest-order contributions from orbitals with principal quantum numbers $n=13-26$ by all-order values [3].

\section{Summary of results}

Following the designations of the Ref. [3], we give a summary of the results for the Stark shift coefficient $k$ in $10^{-10} \mathrm{~Hz} /(\mathrm{V} / \mathrm{m})^{2}$ and the blackbody radiation shift parameter $\beta$. The Stark coefficient $k$ is defined as

$$
\delta \nu=k E^{2}
$$

where $\delta \nu$ is the frequency shift in the static electric field. The Stark coefficient for the transition between states $F$ and $I$ is related to the polarizability as

$$
k=-\frac{1}{2}\left[\alpha_{0}(F)-\alpha_{0}(I)\right]
$$

Theoretical calculations of polarizabilities are generally expressed in atomic units (a.u.), in which, $e, m_{e}, 4 \pi \epsilon_{0}$ and the reduced Planck constant $\hbar$ have the numerical value 1 . The polarizability in a.u. has the dimension of volume, and its numerical values presented here are thus expressed in units of $a_{0}^{3}$, where $a_{0} \approx 0.052918 \mathrm{~nm}$ is the Bohr radius. The atomic units for $\alpha$ can be converted to SI units via where $\left|\Psi_{v}\right\rangle$ and $\left|\Psi_{w}\right\rangle$ are given by the expansion (9). In the SD approximation, the resulting expression for the numerator of Eq. (10) consists of the sum of the DF matrix element $z_{w v}$ and 20 other terms that are linear or quadratic functions of the excitation coefficients. We note that the matrix elements of any one-body operators (for example, electric-dipole and magnetic
hyperfine ones needed for the evaluation of the BBR shifts) can be calculated with the same general code in this approach.
In this approach, numerical wave functions on a radial grid are never explicitly generated; they are represented in terms of the

B. Evaluation of the uncertainties

There are two distinct sources of uncertainties in the eval-
uation of the $\alpha_{F}^{(3)}(0)$ given by Eqs. (4-5):

(1) uncertainty in the values of the individual matrix ele-

(2) uncertainty of the remaining tail contribution.

Stability checks must be carried out to ensure that no significant cancellations are present in the sums that may ad(1) 
TABLE I

SUMMARY OF THE RECENT THEORETICAL CALCULATIONS OF THE STARK SHIFT COEFFICIENT $k$ IN $10^{-10}$ Hz/(V/M) ${ }^{2}$ AND THE BLACKBODY RADIATION SHIFT PARAMETER $\beta$ FOR TRANSITIONS BETWEEN THE GROUND HYPERFINE STATES AND COMPARISON WITH EXPERIMENT.

\begin{tabular}{|l||l||l||c||c||c|}
\hline Atom & Transition & & Ref. & $k$ & $\beta$ \\
\hline${ }^{7} \mathrm{Li}$ & $2 s(F=2 \leftrightarrow F=1)$ & Theory, LCCSD[pT] & {$[26]$} & -0.05824 & $-0.5017 \times 10^{-14}$ \\
& & Experiment & {$[31]$} & $-0.061(2)$ & \\
\hline${ }^{23} \mathrm{Na}$ & $3 s(F=2 \leftrightarrow F=1)$ & Theory, LCCSD[pT] & Present & -0.1285 & $-0.5019 \times 10^{-14}$ \\
& & Experiment & {$[31]$} & $-0.124(3)$ & \\
\hline${ }^{39} \mathrm{~K}$ & $4 s(F=2 \leftrightarrow F=1)$ & Theory, LCCSD[pT] & {$[27]$} & -0.0746 & $-1.118 \times 10^{-14}$ \\
& & Experiment & {$[31]$} & $-0.071(2)$ & \\
\hline${ }^{87} \mathrm{Rb}$ & $5 s(F=2 \leftrightarrow F=1)$ & Theory, LCCSD[pT] & Present, [18] & $-1.272 *$ & $-1.287 \times 10^{-14}$ \\
& & Theory, PTSCI & {$[28]$} & $-1.24(1)$ & $-1.26(1) \times 10^{-14}$ \\
& & Experiment & {$[31]$} & $-1.23(3)$ & \\
\hline${ }^{133} \mathrm{Cs}$ & $6 s(F=4 \leftrightarrow F=3)$ & Theory, LCCSD[pT] & {$[3]$} & $-2.271(8)$ & $-1.710(6) \times 10^{-14}$ \\
& & Theory, PTSCI & {$[2]$} & $-2.26(2)$ & $-1.70(2) \times 10^{-14}$ \\
& & Experiment & {$[4]$} & $-2.271(4)$ & $-1.710(3) \times 10^{-14}$ \\
& & Experiment & {$[32]$} & $-2.05(5)$ & $-1.54(4) \times 10^{-14}$ \\
\hline${ }^{137} \mathrm{Ba}^{+}$ & $6 s(F=2 \leftrightarrow F=1)$ & Theory, PTSCI & {$[28]$} & $-0.284(3)$ & $-0.245(2) \times 10^{-14}$ \\
\hline${ }^{171} \mathrm{Yb}^{+}$ & $6 s(F=1 \leftrightarrow F=0)$ & Theory, RMBPT3 & {$[25]$} & -0.1796 & $-0.0983 \times 10^{-14}$ \\
& & Theory, PTSCI & {$[28]$} & $-0.171(9)$ & $-0.094(5) \times 10^{-14}$ \\
\hline${ }^{199} \mathrm{Hg}^{+}$ & $6 s(F=1 \leftrightarrow F=0)$ & Theory, PTSCI & {$[28]$} & $-0.060(3)$ & $-0.0102(5) \times 10^{-14}$ \\
\hline
\end{tabular}

*Preliminary value

$\alpha / h\left[\mathrm{~Hz} /(V / m)^{2}\right]=2.48832 \times 10^{-8} \alpha[a . u$.$] , where the conver-$ sion coefficient is $4 \pi \epsilon_{0} a_{0}^{3} / h$ and Planck constant $h$ is factored out.

The parameter $\beta$ of the relative temperature-dependent BBR shift of the microwave frequency standard is defined as

$$
\frac{\delta \nu}{\nu_{0}}=\beta\left(\frac{T(K)}{T_{0}}\right)^{4}\left(1+\epsilon\left(\frac{T(K)}{T_{0}}\right)^{2}\right),
$$

where $T_{0}$ is generally taken to be room temperature, $300 \mathrm{~K}$, $\epsilon$ parameterizes the lowest-order (in $\mathrm{T}$ ) contribution to the dynamic correction $\eta$ in Eq. (3), and $\nu_{0}$ is clock transition frequency. The parameter $\beta$ is calculated directly from the Stark-shift coefficient $k$ defined by Eqs. (11-12) as

$$
\beta=\frac{k}{\nu_{0}}(831.9 \mathrm{~V} / \mathrm{m})^{2} .
$$

The small parameter $\epsilon$ has been calculated in Ref. [28] to be equal to 0.011 for ${ }^{87} \mathrm{Rb}, 0.013$ for ${ }^{133} \mathrm{Cs}, 0.004$ for ${ }^{137} \mathrm{Ba}^{+}$, 0.002 for ${ }^{171} \mathrm{Yb}^{+}$, and 0.0005 for ${ }^{199} \mathrm{Hg}^{+}$.

Recent high-precision theoretical results [2], [3], [18], [26], [27], [28] for the Stark shift coefficient $k$ in $10^{-10} \mathrm{~Hz} /(\mathrm{V} / \mathrm{m})^{2}$ and the blackbody radiation shift parameter $\beta$ are summarized in Table I. We use [pT] designation to indicate that valence triple excitations were taken into account where LCCSDpT data were expected to be more accurate that the LCCSD ones. The result for $\mathrm{Yb}^{+}$from Ref. [25] is obtained using relativistic third-order perturbation theory (RMBPT3). Theoretical results are compared with experimental data from Refs. [31], [4], [32]. The most complete comparison with other theory and experimental results for $\mathrm{Cs}$ is given in Ref. [28].

A new preliminary $a b$ initio value of $\beta$ [18] for the ${ }^{87} \mathrm{Rb}$ frequency standards, obtained using the LCCSD[pT] method is presented. We have also evaluated the Stark shift coefficient $k$ and the parameter $\beta$ for ${ }^{23} \mathrm{Na}$ to study how these quantities vary among alkali-metal atoms. We find that the value of $\beta$ for $\mathrm{Na}$ is nearly identical to that for Li and is significantly (by a factor of 3.4) smaller than the value of $\beta$ for Cs.

Theoretical results are in good agreement with each other. We note that both of the approaches contain all-order correlation corrections. However, these methods include somewhat different types of the high-order correlation terms and implementation is quite different. In the PTSCI (or correlation potential) method used in Refs. [2], [28] the calculations start from the relativistic Hartree-Fock method in the $V^{N-1}$ approximation. The correlations are incorporated by means of a correlation potential $\Sigma$. The correlation potential is used to build a new set of single-electron states for subsequent evaluation of the hyperfine and electric-dipole matrix elements using the random-phase approximation. Structural radiation and the normalization corrections are included for hyperfine matrix elements. $\mathrm{Hg}^{+}$and $\mathrm{Yb}^{+}$calculations were carried out with the correlation potential calculated in second-order and scaled to fit the experimental energies. For the other systems, two classes of terms are included in the correlation potential to all orders: screening of the Coulomb interaction and holeparticle interactions. The resulting correlation potential is scaled to fit the experimental energies. Scaling of the all-order correlation potential leads to only small adjustments and serves as a part of the uncertainty evaluation. For $\mathrm{Rb}, \mathrm{Cs}$, and $\mathrm{Ba}^{+}$ scaling of both second-order and all-order correlation potential operator was carried out for additional confirmation of the quoted uncertainties. The assignment of the uncertainty values was also based on the comparison of the polarizabilities and hyperfine constants with experimental values.

The agreement of results in Table I obtained with dis- 
TABLE II

CONTRIBUTIONS TO THE GROUND STATE STATIC SCALAR POLARIZABILITIES IN $\mathrm{CA}^{+}{ }^{+} \mathrm{AND} \mathrm{SR}^{+}$IN UNITS OF $a_{0}^{3}$.

\begin{tabular}{|l||r||l||r|}
\hline $\mathrm{Ca}^{+}$ & $\alpha(4 s)$ & $\mathrm{Sr}^{+}$ & $\alpha(5 s)$ \\
\hline $4 s_{1 / 2}-4 p_{1 / 2}$ & 24.4 & $5 s_{1 / 2}-5 p_{1 / 2}$ & 29.23 \\
$4 s_{1 / 2}-4 p_{3 / 2}$ & 48.4 & $5 s_{1 / 2}-5 p_{3 / 2}$ & 56.48 \\
$4 s_{1 / 2}-(5,6) p_{1 / 2}$ & 0.014 & $5 s_{1 / 2}-(6-8) p_{1 / 2}$ & 0.008 \\
$4 s_{1 / 2}-(5,6) p_{3 / 2}$ & 0.022 & $5 s_{1 / 2}-(6-8) p_{3 / 2}$ & 0.008 \\
\hline$\alpha_{\text {tail }}$ & 0.006 & $\alpha_{\text {tail }}$ & 0.02 \\
$\alpha_{\text {core+vc }}$ & 3.25 & $\alpha_{\text {core }+\mathrm{vc}}$ & 5.55 \\
\hline$\alpha_{\text {total }}$ & 76.1 & $\alpha_{\text {total }}$ & 91.30 \\
\hline
\end{tabular}

tinct high-precision approaches gives another estimate of the accuracy of the theoretical values. The evaluation of the uncertainty of the $\mathrm{Rb} \mathrm{LCCSD}[\mathrm{pT}]$ result is in progress [18]. Table I illustrates the relative size of the electrostatic frequency shifts for the ground state hyperfine transitions in various systems. The ion ${ }^{199} \mathrm{Hg}^{+}$has both the lowest value of $\beta$ and the lowest resulting fractional uncertainty in the frequency standard $\left(5 \times 10^{-18}\right)$ due to BBR shift among the systems listed in Table I.

\section{OPTICAL FREQUENCY STANDARDS WITH $\mathrm{CA}^{+}$AND $\mathrm{SR}^{+}$IONS}

In this section, we discuss the calculation of the BBR shifts for optical frequency standards based on the $4 s-3 d_{5 / 2}$ transition in $\mathrm{Ca}^{+}[19]$ and the $5 s-4 d_{5 / 2}$ transition in $\mathrm{Sr}^{+}$[20]. We note that while the calculations were conducted for ${ }^{43} \mathrm{Ca}^{+}$and ${ }^{88} \mathrm{Sr}^{+}$, we have verified that the results given in this section do not depend on the particular isotope. Therefore, we will omit the $A$ labels in text and tables below. According to Eq. (3), the calculation of the BBR shift for these transitions requires the calculation of the lowest-order atomic polarizability (unlike the cases of the hyperfine transitions, it does not cancel out for optical frequency standards) and the evaluation of the dynamic correction $\eta$. The third-order $F$-dependent polarizability was verified in Ref. [19] to give a negligible contribution to the BBR shift.

Therefore, the evaluation of the BBR shift requires an accurate calculation of static scalar polarizabilities of the $n s_{1 / 2}$ ground and $(n-1) d_{5 / 2}$ excited states. As mentioned earlier, the effect of the tensor part of the $(n-1) d_{5 / 2}$ polarizability is averages out due to the isotropic nature of the electric field radiated by the blackbody.

\section{A. Calculation of the ground state and $n d_{5 / 2}$ state polariz- abilities}

The calculation of the scalar polarizability of a monovalent atom can be separated into three parts: the contribution of the electrons in the ionic core, $\alpha_{\text {core }}$; a small term, $\alpha_{v c}$, that changes the core polarizability due to the presence of the valence electron; and the dominant contribution, $\alpha_{v}$, from the valence electron. The ionic core polarizability used here was calculated using the random-phase approximation (RPA) [36]. We calculate the contribution $\alpha_{v c}$ in the RPA as well
TABLE III

CONTRibutions to THE $3 d_{5 / 2}$ AND $4 d_{5 / 2}$ STATIC SCALAR POLARIZABILITIES IN $\mathrm{CA}^{+} \mathrm{AND} \mathrm{SR}^{+}$, RESPECTIVELY, IN UNITS OF $a_{0}^{3}$.

\begin{tabular}{|l||c||l||c|}
\hline $\mathrm{Ca}^{+}$ & $\alpha\left(3 d_{5 / 2}\right)$ & $\mathrm{Sr}^{+}$ & $\alpha\left(4 d_{5 / 2}\right)$ \\
\hline $3 d_{5 / 2}-4 p_{3 / 2}$ & $22.78(25)$ & $4 d_{5 / 2}-5 p_{3 / 2}$ & $44.16(29)$ \\
$3 d_{5 / 2}-5 p_{3 / 2}$ & $0.011(2)$ & $4 d_{5 / 2}-6 p_{3 / 2}$ & $0.012(2)$ \\
$3 d_{5 / 2}-6 p_{3 / 2}$ & 0.004 & $4 d_{5 / 2}-7 p_{3 / 2}$ & 0.003 \\
\hline $3 d_{5 / 2}-4 f_{5 / 2}$ & $0.120(3)$ & $4 d_{5 / 2}-4 f_{5 / 2}$ & $0.329(4)$ \\
$3 d_{5 / 2}-5 f_{5 / 2}$ & $0.039(2)$ & $4 d_{5 / 2}-5 f_{5 / 2}$ & $0.085(2)$ \\
$3 d_{5 / 2}-6 f_{5 / 2}$ & $0.018(1)$ & $4 d_{5 / 2}-6 f_{5 / 2}$ & 0.035 \\
$3 d_{5 / 2}-(7-12) f_{5 / 2}$ & 0.027 & $4 d_{5 / 2}-(7-12) f_{5 / 2}$ & 0.045 \\
\hline $3 d_{5 / 2}-4 f_{7 / 2}$ & $2.392(53)$ & $4 d_{5 / 2}-4 f_{7 / 2}$ & $6.576(70)$ \\
$3 d_{5 / 2}-5 f_{7 / 2}$ & $0.773(33)$ & $4 d_{5 / 2}-5 f_{7 / 2}$ & $1.699(30)$ \\
$3 d_{5 / 2}-6 f_{7 / 2}$ & $0.350(12)$ & $4 d_{5 / 2}-6 f_{7 / 2}$ & $0.698(11)$ \\
$3 d_{5 / 2}-7 f_{7 / 2}$ & $0.191(7)$ & $4 d_{5 / 2}-7 f_{7 / 2}$ & $0.360(5)$ \\
$3 d_{5 / 2}-8 f_{7 / 2}$ & $0.117(4)$ & $4 d_{5 / 2}-8 f_{7 / 2}$ & $0.212(4)$ \\
$3 d_{5 / 2}-9 f_{7 / 2}$ & $0.077(3)$ & $4 d_{5 / 2}-9 f_{7 / 2}$ & $0.136(2)$ \\
$3 d_{5 / 2}-10 f_{7 / 2}$ & $0.054(2)$ & $4 d_{5 / 2}-10 f_{7 / 2}$ & $0.093(1)$ \\
$3 d_{5 / 2}-11 f_{7 / 2}$ & $0.039(1)$ & $4 d_{5 / 2}-11 f_{7 / 2}$ & $0.067(1)$ \\
$3 d_{5 / 2}-12 f_{7 / 2}$ & $0.029(1)$ & $4 d_{5 / 2}-12 f_{7 / 2}$ & $0.050(1)$ \\
\hline$\alpha_{\text {tail }}$ & $1.7(1.1)$ & $\alpha_{\text {tail }}$ & $2.06(20)$ \\
$\alpha_{\text {core }+\mathrm{vc}}$ & $3.25(17)$ & $\alpha_{\text {core }+\mathrm{vc}}$ & $5.41(31)$ \\
\hline$\alpha_{\text {total }}$ & $32.0(1.1)$ & $\alpha_{\text {total }}$ & $62.0(5)$ \\
\hline
\end{tabular}

for consistency with the ionic core value. The valence scalar polarizability $\alpha_{0}$ of an atom in a state $v$ can be expressed as the sum over all excited intermediate states $n$ allowed by electric-dipole selection rules:

$$
\alpha_{0}=\frac{2}{3\left(2 j_{v}+1\right)} \sum_{n} \frac{\langle n\|D\| v\rangle^{2}}{E_{n}-E_{v}},
$$

where $\langle n\|D\| v\rangle$ are the reduced electric-dipole matrix elements and $E_{i}$ is the energy of the $i$ th state. Just as in the case of the third-order sums in the previous section, the valence polarizability is separated into two parts, the main term containing the first few dominant contributions and the remainder $\alpha_{\text {tail }}$. The matrix elements are calculated using the all-order LCCSD or LCCSDpT approaches described in Section III-A.

Contributions to the ground state static scalar polarizabilities in $\mathrm{Ca}^{+}$and $\mathrm{Sr}^{+}$in units of $a_{0}^{3}$ are listed in Table II. The tail contributions are grouped together as $\alpha_{\text {tail }}$. For the main contribution, we use our $a b$ initio LCCSD all-order values of the matrix elements and experimental energies from Ref. [34]. The first $n s-n p$ transitions contribute over $99.9 \%$ to the valence polarizability.

Contributions to the $3 d_{5 / 2}$ and $4 d_{5 / 2}$ static scalar polarizabilities in $\mathrm{Ca}^{+}$and $\mathrm{Sr}^{+}$, respectively, are listed in Table III. Unlike the case of ground-state polarizabilities, contributions from other excited states are significant for the $n d_{5 / 2}-n^{\prime} f_{7 / 2}$ terms, which converge very slowly. To improve accuracy, contributions with $n>13$ were included in the main term and evaluated using the all-order approach. The evaluation of uncertainties in ground state and $n d_{5 / 2}$ polarizabilities is discussed in the next section. 
TABLE IV

EVALUATION OF THE UNCERTAINTIES OF ELECTRIC-DIPOLE MATRIX ELEMENTS IMPORTANT FOR BBR SHIFT CALCULATION IN CA ${ }^{+}$AND SR $^{+}$.

ABSOLUTE VALUES IN A.U. ARE GIVEN.

\begin{tabular}{|l||l||c||c||c||c||c|}
\hline Atom & Transition & LCCSD & LCCSD $_{s c}$ & LCCSDpT & LCCSDpT $_{s c}$ & Final \\
\hline $\mathrm{Ca}^{+}$ & $3 d_{5 / 2}-4 p_{3 / 2}$ & 3.245 & 3.306 & 3.313 & 3.288 & $3.306(18)$ \\
& $3 d_{5 / 2}-4 f_{5 / 2}$ & 0.501 & 0.516 & 0.517 & 0.511 & $0.516(6)$ \\
& $3 d_{5 / 2}-4 f_{7 / 2}$ & 2.238 & 2.309 & 2.310 & 2.284 & $2.309(25)$ \\
\hline \multirow{2}{*}{$\mathrm{Sr}^{+}$} & $4 d_{5 / 2}-5 p_{3 / 2}$ & 4.150 & 4.187 & 4.198 & 4.173 & $4.187(14)$ \\
& $4 d_{5 / 2}-4 f_{5 / 2}$ & 0.779 & 0.789 & 0.790 & 0.785 & $0.789(4)$ \\
& $4 d_{5 / 2}-4 f_{7 / 2}$ & 3.486 & 3.528 & 3.536 & 3.509 & $3.528(19)$ \\
& $4 d_{3 / 2}-5 p_{1 / 2}$ & 3.083 & 3.112 & 3.119 & 3.102 & $3.112(10)$ \\
& $4 d_{3 / 2}-5 p_{3 / 2}$ & 1.369 & 1.383 & 1.386 & 1.378 & $1.383(5)$ \\
\hline
\end{tabular}

\section{B. Evaluation of the uncertainties}

There are three sources of the uncertainties contributing to polarizabilities of $\mathrm{Ca}^{+}$and $\mathrm{Sr}^{+}$in the ground and $n d_{5 / 2}$ states. The ionic core contribution taken from Ref. [36] is relatively small and is expected to be accurate to better than $5 \%$, based on the comparison of RPA and experimental polarizabilities for noble gases. Its uncertainty is, however, irrelevant to the evaluation of the uncertainty of the BBR shift since the core contribution is identical for both clock states. The small compensating term $\alpha_{v c}$ is different for the two states, but its entire contribution is below the present uncertainty of the other terms.

(1) Ground-state polarizability. The uncertainties in the values of the $4 s-4 p_{1 / 2,3 / 2}$ and the $5 s-5 p_{1 / 2,3 / 2}$ matrix elements for $\mathrm{Ca}^{+}$and $\mathrm{Sr}^{+}$, respectively, completely determine the uncertainty in the valence ground state polarizability values since they contribute over $99.9 \%$. The LCCSD values for the primary $n s-n p$ transitions in $\mathrm{Li}, \mathrm{Na}, \mathrm{K}, \mathrm{Rb}$, and $\mathrm{Cs}$ agree with various types of high-precision experiments to $0.1 \%-0.4 \%$ [33]. There is no reason to expect reduced theoretical accuracy in the cases of either $\mathrm{Ca}^{+}$or $\mathrm{Sr}^{+}$, and $0.5 \%$ uncertainty was assigned to the $5 s-5 p_{1 / 2}$ and $5 s-5 p_{3 / 2} \mathrm{Sr}^{+}$matrix elements [20]. The resulting uncertainty of the $5 s$ polarizability is $1 \%$. Our theoretical LCCSD $5 p_{1 / 2,3 / 2}$ lifetimes [20] are in agreement with $1 \%$ experiment conducted in 1995 [37]. The contribution of the $5 s-5 p$ transitions to the lifetimes is dominant $(94 \%)$. However, the theoretical lifetimes of the $\mathrm{Ca}^{+} 4 p_{1 / 2,3 / 2}$ states calculated by exactly the same approach in [19] are in significant (over 3\%) disagreement with 1993 $0.3 \%$ measurement [38]. This issue is discussed in detail in Ref. [19]. Unfortunately, we know of no way to accurately estimate the missing additional contributions to the dominant correlation correction to these transitions as it can be done for some other transitions (see the discussion below). Accurate new measurements of the $n p$ lifetimes or $n s-n p$ oscillator strengths in $\mathrm{Ca}^{+}$and $\mathrm{Sr}^{+}$are needed to help resolve this issue and improve the final BBR shift results.

(2) Excited $n d_{5 / 2}$ state polarizability: the main term. The uncertainty in the main term of the $n d_{5 / 2}$ state polarizability is dominated by a very few terms as illustrated by Table III. We obtain accurate values for these matrix elements using a semi-empirical scaling procedure that evaluates some classes of correlation corrections omitted by the current all-order calculations. The scaling procedure is described in Ref. [29]. Briefly, the single valence excitation coefficients are multiplied by the ratio of the corresponding experimental and theoretical correlation energies, and the matrix element calculation is repeated with the modified excitation coefficients. The scaling procedure is particularly suitable for these transitions because the matrix element contribution containing the single valence excitation coefficients is dominant in these cases (but not for the primary $n s-n p$ matrix elements discussed earlier). We note that this procedure is equivalent to scaling of the correlation potential in the PTSCI approach discussed in Section III-C. We conduct scaling starting from both LCCSD and LCCSDpT approximations. The scaling factors for the LCCSD and LCCSDpT calculations are different, and we take scaled LCCSD values to be our final results based on comparisons of similar calculations in alkali-metal atoms with experiments [39], [40], [41]. The uncertainty evaluation of the reduced matrix elements that give significant contributions to the polarizabilities is illustrated in Table IV, scaled values are listed with subscript "sc". The uncertainties quoted in the table are taken to be the maximum difference between the scaled LCCSD values and the $a b$ initio LCCSDpT and scaled LCCSDpT values. A notable feature of this table is close agreement of the scaled LCCSD and LCCSDpT results.

(3) Excited $n d_{5 / 2}$ state polarizability: the tail term. The tail contribution of the $n d_{5 / 2}-n^{\prime} f_{7 / 2}$ terms is particularly large; its DF value (3.5 a.u.) is $5 \%$ of the total polarizability for $\mathrm{Sr}^{+}$. The uncertainty in the tail dominates the uncertainty of the $\mathrm{Ca}^{+}$BBR shift value calculated in Ref. [19]. In a later work on $\mathrm{Sr}^{+}$, this issue was resolved by performing additional RPA calculations of the tail and carrying out scaling procedures starting from both DF and RPA approximations. Since the largest part of the correlation correction for the $4 d_{5 / 2}-n f_{7 / 2}$ transitions with $n>9$ comes from RPAlike terms, the RPA approximation is expected to produce a better result than the DF one. We carried out the RPA calculation of the tail and obtained a lower value of 2.9 a.u. We also calculated the main terms using the DF and RPA approximations and compared the results with our all-order values. Contributions to scalar polarizability of the $4 d_{5 / 2}$ state in $\mathrm{Sr}^{+}$from terms involving $4 d_{5 / 2}-n f_{7 / 2}$ transitions with $n=4-12$ calculated in the DF, RPA, and all-order approximations are listed in Table $\mathrm{V}$. The relative differences 
TABLE V

CONTRIBUTIONS TO THE $4 d_{5 / 2}$ STATIC SCALAR POLARIZABILITIES IN $\mathrm{SR}^{+}$FROM $n f_{7 / 2}$ STATES IN DF, RPA, AND ALL-ORDER APPROXIMATIONS IN UNITS OF $a_{0}^{3}$. THE RELATIVE DIFFERENCES BETWEEN DF AND ALL-ORDER RESULTS AND BETWEEN RPA AND ALL-ORDER RESULTS ARE LISTED IN \% IN THE LAST TWO COLUMNS.

\begin{tabular}{|l||c||c||c||c||c|}
\hline & DF & RPA & All-order & $\Delta(\mathrm{DF})$ & $\Delta(\mathrm{RPA})$ \\
\hline $4 d_{5 / 2}-4 f_{7 / 2}$ & 11.427 & 10.903 & 6.576 & 42.46 & 39.69 \\
$4 d_{5 / 2}-5 f_{7 / 2}$ & 2.725 & 2.545 & 1.698 & 37.66 & 33.26 \\
$4 d_{5 / 2}-6 f_{7 / 2}$ & 1.089 & 1.004 & 0.698 & 35.91 & 30.51 \\
$4 d_{5 / 2}-7 f_{7 / 2}$ & 0.556 & 0.509 & 0.359 & 35.33 & 29.32 \\
$4 d_{5 / 2}-8 f_{7 / 2}$ & 0.327 & 0.297 & 0.212 & 35.10 & 28.70 \\
$4 d_{5 / 2}-9 f_{7 / 2}$ & 0.210 & 0.191 & 0.136 & 35.08 & 28.43 \\
$4 d_{5 / 2}-10 f_{7 / 2}$ & 0.144 & 0.130 & 0.093 & 35.09 & 28.25 \\
$4 d_{5 / 2}-11 f_{7 / 2}$ & 0.103 & 0.093 & 0.067 & 35.10 & 28.14 \\
$4 d_{5 / 2}-12 f_{7 / 2}$ & 0.077 & 0.069 & 0.050 & 35.15 & 28.03 \\
\hline Tail & 3.48 & 2.86 & & & \\
Adjusted tail & 2.26 & 2.06 & & & \\
\hline
\end{tabular}

between DF and all-order results, and between RPA and all-order results, are listed in $\%$ in columns labeled $\Delta$ (DF) and $\Delta$ (RPA), respectively. The DF and RPA approximations overestimate the polarizability contributions by $35 \%$ and $28 \%$, respectively. To improve our accuracy, we scale both DF and RPA results for the tail by these respective amounts to obtain a DF-scaled value of 2.26 a.u. and RPA-scaled value of 2.06 a.u. We take the RPA-scaled value to be the final one and the difference between these two values to be its uncertainty.

\section{Results for the BBR shifts}

The scalar polarizability values are used to evaluate the BBR shifts in the $\mathrm{Ca}^{+}$and $\mathrm{Sr}^{+}$clock transitions at $T=300 \mathrm{~K}$ using Eq. (3) [19], [20]. The dynamic correction was estimated for $\mathrm{Sr}^{+}$to be $\eta=0.0013$ and $\eta=0.0064$ for the $5 s$ and $4 d_{3 / 2}$ states, respectively, using formulas from [24]. The resulting correction to $\mathrm{Sr}^{+} \mathrm{BBR}$ shift is $-0.002 \mathrm{~Hz}$ and the final value for the BBR shift is $0.250(9) \mathrm{Hz}$ [20]. The M1 and E2 contributions to the $\mathrm{Sr}^{+}$BBR shift were evaluated in [20] using the approach described in Ref. [24] and found to be negligible (below $0.01 \%$ ). Comparisons of LCCSD[pT] BBR shift values with other theoretical results are given in Ref. [19] and Ref. [20], for $\mathrm{Ca}^{+}$and $\mathrm{Sr}^{+}$, respectively.

Results for the BBR shift $\delta \nu$ at $T=300 K$, the corresponding fractional uncertainties $\delta \nu / \nu_{0}$, and the fractional error in the absolute transition frequency induced by the BBR shift uncertainties are listed in Table VI. We note that the relative uncertainty in the BBR shift is significantly larger than the polarizability uncertainties owing to the cancellation between the values of the polarizabilities of the clock states.

Further improvement in the BBR shift values will require settling the issue of the accuracy of the primary $n s-n p$ matrix elements as well as experimental determination of the $n p_{3 / 2}-(n-1) d_{5 / 2}$ matrix elements, where $n s$ is the ground state. The types of experiments that can provide better understanding of the theoretical uncertainties or extraction of
TABLE VI

THE BBR SHIFT $\delta \nu$ AT $T=300 K$, CLOCK TRANSITION FREQUENCY $\nu_{0}$, FRACTIONAL UNCERTAINTIES $\delta \nu / \nu_{0}$ DUE TO BBR SHIFT, AND THE FRACTIONAL ERROR IN THE ABSOLUTE TRANSITION FREQUENCY INDUCED BY THE BBR SHIFT UNCERTAINTY.

\begin{tabular}{|l||c||c||c||c|}
\hline Ion & $\delta \nu(\mathrm{Hz})$ & $\nu_{0}(\mathrm{~Hz})$ & $\delta \nu / \nu_{0}$ & Uncertainty \\
\hline $\mathrm{Ca}^{+}$ & $0.38(1)[19]$ & $4.11 \times 10^{14}$ & $9.24 \times 10^{-16}$ & $2.4 \times 10^{-17}$ \\
$\mathrm{Sr}^{+}$ & $0.250(9)[20]$ & $4.45 \times 10^{14}$ & $5.62 \times 10^{-16}$ & $2.0 \times 10^{-17}$ \\
\hline
\end{tabular}

the specific matrix elements for more accurate evaluation of the BBR shifts include $n p$ lifetime (or $n s-n p$ oscillator strength) measurements, light shift ratio measurements [42], or ac or dc Stark shift measurements on any of the lines involving $n s, n p$, or $(n-1) d$ states. We estimate that the experimental determination of the $n s-n p_{1 / 2,3 / 2}$ matrix elements, where $n s$ is a ground state accurate to $0.15 \%$ (or $0.3 \% n p_{1 / 2,3 / 2}$ lifetime measurement) would reduce fractional error in the absolute transition frequency induced by the BBR shift uncertainty by a factor of 2 .

\section{OPTICAL FREQUENCY STANDARDS WITH DIVALENT ATOMS}

BBR shifts for optical lattice clocks based on the $n s^{2}{ }^{1} S_{0}-$ nsnp ${ }^{3} P_{0}$ transitions in divalent atoms were calculated for $\mathrm{Mg}, \mathrm{Ca}, \mathrm{Sr}$, and $\mathrm{Yb}$ in Ref. [24] and for $\mathrm{Hg}$ in Ref. [43]. The evaluation of the BBR shift in these systems requires evaluation of the ground and $n s n p{ }^{3} P_{0}$ state polarizabilities. Different theoretical methods are required for the evaluation of these polarizabilities in comparison with the monovalent systems discussed in the previous sections. The CI + MBPT approach initially developed in Ref. [44] that combines the configuration-interaction (CI) method and perturbation theory was used in Refs. [24], [43]. Experimental data were used where available for dominant contributions. The Sr BBR shift was later investigated in more detail in Ref. [45] using the same approach.

In the CI method, the many-electron wave function is obtained as a linear combination of all distinct states of a given angular momentum $\mathbf{J}$ and parity:

$$
\Psi_{J}=\sum_{i} c_{i} \Phi_{i}
$$

in other words, a linear combination of Slater determinants of a proper symmetry from a model subspace [44].

Energies and wave functions of low-lying states are determined by diagonalizing an effective Hamiltonian:

$$
H^{\text {eff }}=H_{1}+H_{2}
$$

where $H_{1}$ represents the one-body part of the Hamiltonian, and the two-body part $\mathrm{H}_{2}$ contains the Coulomb (or Coulomb + Breit) matrix elements $v_{i j k l}$. The resulting wave functions are used to calculate matrix elements and other properties.

The CI + MBPT approach permits one to incorporate core excitations in the CI method by including certain perturbation theory terms into an effective Hamiltonian (17). The one-body 
TABLE VII

COMPARISON OF THE CI + ALL-ORDER RESULTS FOR THE ENERGY LEVELS OF SR WITH EXPERIMENT. TWO-ELECTRON BINDING ENERGY IS GIVEN IN THE FIRST ROW, THE OTHER VALUES ARE COUNTED FROM THE GROUND STATE. THE ENERGIES ARE GIVEN IN $\mathrm{CM}^{-1}$. THE RELATIVE DIFFERENCE WITH EXPERIMENTAL VALUES IS GIVEN IN THE LAST COLUMN IN \%.

\begin{tabular}{|l||c||c||c|}
\hline Level & Expt. & Present & $\Delta(\%)$ \\
\hline $5 s^{2}{ }^{1} S_{0}$ & 134896 & 134894 & 0.001 \\
\hline $5 s 5 p^{3} P_{0}$ & 14318 & 14301 & 0.11 \\
$5 s 5 p^{3} P_{1}$ & 14504 & 14487 & 0.12 \\
$5 s 5 p^{3} P_{2}$ & 14899 & 14892 & 0.04 \\
\hline $5 s 4 d^{3} D_{1}$ & 18159 & 18148 & 0.06 \\
$5 s 4 d^{3} D_{2}$ & 18219 & 18218 & 0.01 \\
$5 s 4 d^{3} D_{3}$ & 18319 & 18335 & -0.09 \\
\hline $5 s 4 d^{1} D_{2}$ & 20150 & 20222 & -0.36 \\
$5 s 5 p^{1} P_{1}$ & 21698 & 21746 & -0.22 \\
$5 s 6 s^{3} S_{1}$ & 29039 & 29090 & -0.18 \\
$5 s 6 s^{1} S_{0}$ & 30592 & 30656 & -0.21 \\
\hline $4 d 5 p^{3} F_{2}$ & 33267 & 33389 & -0.37 \\
$4 d 5 p^{3} F_{3}$ & 33590 & 33756 & -0.49 \\
$4 d 5 p^{3} F_{4}$ & 33919 & 34110 & -0.56 \\
\hline $4 d 5 p^{1} D_{2}$ & 33827 & 34005 & -0.53 \\
\hline $5 s 6 p^{3} P_{0}$ & 33854 & 33881 & -0.08 \\
$5 s 6 p^{3} P_{1}$ & 33868 & 33894 & -0.08 \\
$5 s 6 p^{3} P_{2}$ & 33973 & 33915 & 0.17 \\
\hline $5 s 6 p^{1} P_{1}$ & 34098 & 34138 & -0.12 \\
$5 s 5 d^{1} D_{2}$ & 34727 & 34777 & -0.14 \\
\hline$\Delta\left(^{3} D_{1}-{ }^{3} P_{0}\right)$ & 3842 & 3847 & 0.14 \\
\hline
\end{tabular}

part $H_{1}$ is modified to include the correlation potential $\Sigma_{1}$ that accounts for part of the core-valence correlations,

$$
H_{1} \rightarrow H_{1}+\Sigma_{1} \text {. }
$$

Either the second-order expression, $\Sigma_{1}^{(2)}$, or all-order chains of such terms can be used (see, for example, Ref. [46]). The twobody Coulomb interaction term $\mathrm{H}_{2}$ is modified by including the two-body part of core-valence interaction that represents screening of the Coulomb interaction by valence electrons;

$$
H_{2} \rightarrow H_{2}+\Sigma_{2},
$$

where $\Sigma_{2}$ is calculated using second-order MBPT in the CI+MBPT approach. The CI method is then applied as usual with the modified $H^{\text {eff }}$ to obtain improved energies and wave functions. The estimated accuracy of BBR values calculated using this approach was $2.7 \%$ for $\mathrm{Mg}, 1.4 \%$ for $\mathrm{Ca}$ and $\mathrm{Sr}$, and $10 \%$ for $\mathrm{Yb}$ [24]. The resulting fractional uncertainties in the clock transition frequencies ranged from $1 \times 10^{-17}$ for $\mathrm{Mg}$ to $3 \times 10^{-16}$ for $\mathrm{Yb}$. The development of a more accurate approach is needed for further improvement of the BBR values for the optical lattice clocks.

\section{A. CI+ all-order method}

The MBPT corrections associated with terms $\Sigma_{1}$ in Eq. (18) and $\Sigma_{2}$ in Eq. (19) typically grow with nuclear charge $Z$. This leads to a deterioration in the accuracy of the $\mathrm{CI}+$ second order MBPT results for heavier, more complicated systems (as illustrated by significantly reduced accuracy of the Yb BBR in Ref. [24]). The order-by-order extension of this method does not look promising as the complexity of the MBPT expansion for systems with more than one valence electron makes a third-order extension impractical. Moreover, although the convergence of the MBPT series is not well studied, it is known that third-order results are often less accurate than the second-order ones. This is why it is important to develop an all-order extension of the CI + MBPT method.

In the CI + all-order approach, introduced in Ref. [21], corrections to the effective Hamiltonian $\Sigma_{1}$ and $\Sigma_{2}$ are calculated using a modified version of the LCCSD all-order method described in Section III-A. Therefore, the effective Hamiltonian contains dominant core and core-valence correlation corrections to all orders. The core-core and core-valence sectors of the correlation corrections for systems with few valence electrons are treated in the all-order method with the same accuracy as in the all-order approach for the monovalent systems. The CI method is then used to treat valence-valence correlations.

The CI + all-order approach is based on the BrilloiunWigner variant of the many-body perturbation theory, rather than the Rayleigh-Schrödinger variant. Use of the RayleighSchrödinger MBPT for systems with more than one valence electron leads to a non-symmetric effective Hamiltonian and to the problem of "intruder states". In the Brilloiun-Wigner variant of MBPT, the effective Hamiltonian is symmetric and accidentally small denominators do not arise; however, $\Sigma_{1}$ and $\Sigma_{2}$ became energy dependent (see Ref. [21] for the formulas and detail description of the CI + all-order method).

This approach has been tested on the calculation of energy levels of $\mathrm{Mg}, \mathrm{Ca}, \mathrm{Sr}, \mathrm{Zn}, \mathrm{Cd}, \mathrm{Ba}$, and $\mathrm{Hg}$ in Ref. [21]. The CI+ all-order method described above treats electronic correlation in systems with several valence electrons in a significantly more complete way than the CI + MBPT approach owing to the inclusion of the additional classes of MBPT terms in $\Sigma_{1}$ and addition of all-order (rather than second-order) correction in $\Sigma_{2}$. We also find almost no deterioration in accuracy of the two-electron binding energies from $\mathrm{Ca}$ to $\mathrm{Hg}$ with $\mathrm{CI}+$ all-order method. At least factor of three improvement in agreement with experimental values for the two-electron binding energies and most excited state energies in comparison with the CI + MBPT method was found even when completely $a b$ initio version of the method was used. Theoretical values for energies are brought into very close agreement with experiment when the energy dependence of $\Sigma\left(\tilde{\epsilon}_{v}\right)$ is used to further improve the wave functions for subsequent use in the polarizability calculations. The results for $\mathrm{Sr}$ energy levels calculated by the $\mathrm{CI}+$ all-order method with adjusted $\tilde{\epsilon}_{v}$ are listed in Table VII for illustration of the $\mathrm{CI}+$ all-order approach. Two-electron binding energies are given in the first row, energies in other rows are counted from the ground state. The energies are given in $\mathrm{cm}^{-1}$ and the relative differences with experimental values are given in the last column in $\%$.

Our preliminary calculations of the ${ }^{3} P_{0}$ polarizability values in $\mathrm{Ca}$ and $\mathrm{Sr}$ indicate better agreement of the $\mathrm{CI}+$ all-order $a b$ initio results with recommended values from Ref. [24] in 
TABLE VIII

SUMMARY OF THE FRACTIONAL UNCERTAINTIES $\delta \nu / \nu_{0}$ DUE TO BBR SHIFT AND THE FRACTIONAL ERROR IN THE ABSOLUTE TRANSITION FREQUENCY INDUCED BY THE BBR SHIFT UNCERTAINTY AT $T=300 \mathrm{~K}$ IN VARIOUS FREQUENCY STANDARDS.

\begin{tabular}{|c|c|c|c|c|}
\hline Atom & Clock transition & Ref. & $\delta \nu / \nu_{0}$ & Uncertainty \\
\hline${ }^{87} \mathrm{Rb}$ & $5 s(F=2-F=1)$ & {$[28]$} & $-1.25 \times 10^{-14}$ & $1 \times 10^{-16}$ \\
${ }^{133} \mathrm{Cs}$ & $6 s(F=4-F=3)$ & {$[3]$} & $-1.732 \times 10^{-14}$ & $6 \times 10^{-17}$ \\
& & {$[2]$} & $-1.72 \times 10^{-14}$ & $2 \times 10^{-16}$ \\
& & {$[4]$} & $-1.732 \times 10^{-14}$ & $3 \times 10^{-17}$ \\
${ }^{137} \mathrm{Ba}^{+}$ & $6 s(F=2-F=1)$ & {$[28]$} & $-2.46 \times 10^{-15}$ & $2 \times 10^{-17}$ \\
${ }^{171} \mathrm{Yb}^{+}$ & $6 s(F=1-F=0)$ & {$[28]$} & $-9.4 \times 10^{-16}$ & $5 \times 10^{-17}$ \\
${ }^{199} \mathrm{Hg}^{+}$ & $6 s(F=1-F=0)$ & {$[28]$} & $-1.02 \times 10^{-16}$ & $5 \times 10^{-18}$ \\
\hline $\mathrm{Ca}^{+}$ & $4 s-3 d_{5 / 2}$ & {$[19]$} & $9.24 \times 10^{-16}$ & $2 \times 10^{-17}$ \\
$\mathrm{Sr}^{+}$ & $5 s-4 d_{5 / 2}$ & {$[20]$} & $5.62 \times 10^{-16}$ & $2 \times 10^{-17}$ \\
\hline $\mathrm{Mg}$ & $3 s^{2}{ }^{1} S_{0}-3 s 3 p^{3} P_{0}$ & {$[24]$} & $-3.9 \times 10^{-16}$ & $1 \times 10^{-17}$ \\
$\mathrm{Al}{ }^{+}$ & $3 s^{2}{ }^{1} S_{0}-3 s 3 p^{3} P_{0}$ & {$[47]$} & $-3.8 \times 10^{-18}$ & $3 \times 10^{-18}$ \\
$\mathrm{Ca}$ & $4 s^{2}{ }^{1} S_{0}-4 s 4 p^{3} P_{0}$ & {$[24]$} & $-2.6 \times 10^{-15}$ & $4 \times 10^{-17}$ \\
$\mathrm{Sr}$ & $5 s^{2}{ }^{1} S_{0}-5 s 5 p^{3} P_{0}$ & {$[24]$} & $-5.5 \times 10^{-15}$ & $7 \times 10^{-17}$ \\
$\mathrm{Yb}$ & $6 s^{2}{ }^{1} S_{0}-6 s 6 p^{3} P_{0}$ & {$[24]$} & $-2.6 \times 10^{-15}$ & $3 \times 10^{-16}$ \\
$\mathrm{Hg}$ & $6 s^{2}{ }^{1} S_{0}-6 s 6 p^{3} P_{0}$ & {$[43]$} & $-1.6 \times 10^{-16}$ & \\
\hline $\mathrm{Ag}$ & $4 d^{10} 5 s^{2} S_{1 / 2}-4 d^{9} 5 s^{2}{ }^{2} D_{5 / 2}$ & {$[48]$} & $-1.9 \times 10^{-16}$ & \\
$\mathrm{Yb}+$ & $4 f^{14} 6 s^{2} S_{1 / 2}-4 f^{13} 6 s^{2}{ }^{2} F_{7 / 2}$ & {$[49]$} & $-2.5 \times 10^{-16}$ & $1 \times 10^{-16}$ \\
\hline
\end{tabular}

comparison with the CI+MBPT approach.

Our further development of this method will include addition of the all-order terms beyond RPA to the treatment of the transition matrix elements for precision calculation of $\mathrm{BBR}$ shifts in divalent systems.

\section{SUMMARY AND CONCLUSION}

In this work, we have presented a review of the most recent high-precision $a b$ initio theoretical calculations of the blackbody radiation (BBR) shifts in various systems of interest to atomic clock research. A recently developed method that combines the relativistic all-order method and the configuration interaction method for accurate calculations of BBR shifts in divalent systems such as $\mathrm{Sr}$ is discussed. High-precision methods used in the recent calculations of the BBR shifts are described. The evaluation of uncertainties in theoretical BBR shifts is discussed in detail. Results for fractional uncertainties $\delta \nu / \nu_{0}$ due to BBR shifts and the fractional error in the absolute transition frequencies induced by the BBR shift uncertainty at $T=300 K$ in various frequency standards are summarized in Table VIII. As illustrated by Table VIII, the fractional errors in absolute transition frequencies induced by uncertainties in theoretical values of the BBR shift are still large for most of the optical frequency standards. Both new experiments and improvement of the theoretical accuracy will be needed to reduce these uncertainties to $10^{-18}$ level.

\section{ACKNOWLEDGMENT}

Part of this research was performed under the sponsorship of the US Department of Commerce, National Institute of Standards and Technology. This work was supported in part by US National Science Foundation Grant No. PHY-07-58088 and by the RFBR grant No. 08-02-00460. MGK thanks University of Delaware for hospitality.

\section{REFERENCES}

[1] M. Lombardi, T. Heavner, and S. Jefferts, "NIST primary frequency standards and the realization of the SI second," Measure: The Journal of Measurement Science, vol. 2, p. 74, 2007.

[2] E. J. Angstmann, V. A. Dzuba, and V. Flambaum, "Frequency shift of the cesium clock transition due to blackbody radiation," Phys. Rev.Lett., vol. 97, p. 040802, 2006.

[3] K. Beloy, U. I. Safronova, and A. Derevianko, "High-accuracy calculation of the blackbody radiation shift in the ${ }^{133} \mathrm{Cs}$ primary frequency standard," Phys. Rev.Lett., vol. 97, p. 040801, 2006.

[4] E. Simon, P. Laurent, and A. Clairon, "Measurement of the stark shift of the Cs hyperfine splitting in an atomic fountain," Phys. Rev. A, vol. 57, p. 426, 1998.

[5] International Committee for Weights and Measures, Proceedings of the sessions of the 95th meeting (October 2006), URL=http://www.bipm.org/utils/en/pdf/CIPM2006-EN.pdf.

[6] A. Godone, F. Levi, S. Micalizio, E. K. Bertacco, and C. E. Calosso, "Frequency-stability performances of the pulsed optically pumped rubidium clock: recent results and future perspectives," IEEE Transactions on Instrumentation and Measurement, vol. 56, p. 378, 2007.

[7] J. Vanier and C. Mandache, "The passive optically pumped Rb frequency standard: the laser approach," Appl. Phys. B, vol. 87, p. 565, 2007.

[8] A. A. Madej, J. E. Bernard, P. Dube, and L. Marmet, "Absolute frequency of the ${ }^{88} \mathrm{Sr}^{+} 5 s^{2} S_{1 / 2} \rightarrow 4 d^{2} D_{5 / 2}$ reference transition at $445 \mathrm{THz}$ and evaluation of systematic shifts," Phys. Rev. A, vol. 70, p. 012507, 2004.

[9] H. S. Margolis, G. P. Barwood, G. Huang, H. A. Klein, S. N. Lea, K. Szymaniec, and P. Gill, "Hertz-level measurement of the optical clock frequency in a single ${ }^{88} \mathrm{Sr}^{+}$ion," Science, vol. 306, p. 19, 2004.

[10] J. E. Stalnaker, S. A. Diddams, T. M. Fortier, K. Kim, L. Hollberg, J. C. Bergquist, W. M. Itano, M. J. Delany, L. Lorini, W. H. Oskay, T. P. Heavner, S. R. Jefferts, F. Levi, T. E. Parker, and J. Shirley, "Optical-tomicrowave frequency comparison with fractional uncertainty of $10^{-15}$," Appl. Phys. B, vol. 89, p. 167, 2007.

[11] W. H. Oskay, S. A. Diddams, E. A. Donley, T. M. Fortier, T. P. Heavner, L. Hollberg, W. M. Itano, S. R. Jefferts, M. J. Delaney, K. Kim, F. Levi, T. E. Parker, and J. C. Bergquist, "Single-atom optical clock with high accuracy,” Phys. Rev. Lett., vol. 97, p. 020801, 2006. 
[12] J. Stenger, C. Tamm, N. Haverkamp, S. Weyers, and H. R. Telle, "Absolute frequency measurement of the $435.5-\mathrm{nm}{ }^{171} \mathrm{Yb}^{+}$-clock transition with a kerr-lens mode-locked femtosecond laser," Opt. Lett., vol. 26, p. $1589,2001$.

[13] P. J. Blythe, S. A. Webster, H. S. Margolis, S. N. Lea, G. Huang, S.K. Choi, W. R. C. Rowley, P. Gill, and R. S. Windeler, "Subkilohertz absolute-frequency measurement of the 467-nm electric octupole transition in ${ }^{171} \mathrm{Yb}+, "$ Phys. Rev. A, vol. 67, p. 020501, 2003.

[14] G. K. Campbell, A. D. Ludlow, S. Blatt, J. W. Thomsen, M. J. Martin, M. H. G. de Miranda, T. Zelevinsky, M. M. Boyd, J. Ye, S. A. Diddams, T. P. Heavner, T. E. Parker, and S. R. Jefferts, "The absolute frequency of the ${ }^{87} \mathrm{Sr}$ optical clock transition," Metrologia, vol. 45, pp. 539-548, 2008.

[15] X. Baillard, M. Fouché, R. L. Targat, P. G. Westergaard, A. Lecallier, F. Chapelet, M. Abgrall, G. D. Rovera, P. Laurent, P. Rosenbusch, S. Bize, G. Santarelli, A. Clairon, P. Lemonde, G. Grosche, B. Lipphardt, and $\mathrm{H}$. Schnatz, "An optical lattice clock with spin-polarized ${ }^{87} \mathrm{Sr}$ atoms," Eur. Phys. J. D, vol. 48, p. 11, 2008.

[16] M. M. Boyd, A. D. Ludlow, S. Blatt, S. M. Foreman, T. Ido, T. Zelevinsky, and J. Ye, " $87 \mathrm{Sr}$ lattice clock with inaccuracy below $10^{-15}$," Phys. Rev. Lett., vol. 98, p. 083002, 2007.

[17] S. A. Diddams, T. Udem, J. C. Bergquist, E. A. Curtis, R. E. Drullinger, L. Hollberg, W. M. Itano, W. D. Lee, C. W. Oates, K. R. Vogel, and D. J. Wineland, "An optical clock based on a single trapped ${ }^{199} \mathrm{Hg}^{+}$ ion," Science, vol. 293, p. 825, 2001.

[18] M. S. Safronova and U. I. Safronova, "Blackbody-radiation shift in the rubidium frequency standard," to be submitted to Phys. Rev. A (2009).

[19] B. Arora, M. S. Safronova, and C. W. Clark, "Blackbody-radiation shift in a ${ }^{43} \mathrm{Ca}^{+}$ion optical frequency standard," Phys. Rev. A, vol. 76, p. 064501, 2007.

[20] D. Jiang, B. Arora, M. S. Safronova, and C. W. Clark, "Blackbodyradiation shift in a ${ }^{88} \mathrm{Sr}^{+}$ion optical frequency standard," arXiv:0904.2107, accepted for publication to J. Phys. B (2009).

[21] M. S. Safronova, M. G. Kozlov, W. R. Johnson, and D. Jiang, "Development of the configuration-interaction + all-order method for atomic calculations," to be submitted to Phys. Rev. A (2009).

[22] J. W. Farley and W. H. Wing, "Accurate calculation of dynamic stark shifts and depopulation rates of Rydberg energy levels induced by blackbody radiation. Hydrogen, helium, and alkali-metal atoms," Phys. Rev. A, vol. 23, p. 2397, 1981.

[23] W. M. Itano, L. L. Lewis, and D. J. Wineland, "Shift of ${ }^{2} s_{1 / 2}$ hyperfine splittings due to blackbody radiation," Phys. Rev. A, vol. 25, p. 1233 , 1982.

[24] S. G. Porsev and A. Derevianko, "Multipolar theory of blackbody radiation shift of atomic energy levels and its implications for optical lattice clocks," Phys. Rev. A, vol. 74, p. 020502(R), 2006.

[25] U. I. Safronova and M. S. Safronova, "Third-order relativistic manybody calculations of energies, transition rates, hyperfine constants, and blackbody radiation shift in ${ }^{171} \mathrm{Yb}^{+}$," Phys. Rev. A, vol. 79, p. 022512, 2009.

[26] W. R. Johnson, U. I. Safronova, A. Derevianko, and M. S. Safronova, "Relativistic many-body calculation of energies, lifetimes, hyperfine constants, and polarizabilities in ${ }^{7} \mathrm{Li}$," Phys. Rev. A, vol. 77, p. 022510 , 2008.

[27] U. I. Safronova and M. S. Safronova, "High-accuracy calculation of energies, lifetimes, hyperfine constants, multipole polarizabilities, and blackbody radiation shift in ${ }^{39} \mathrm{~K}$," Phys. Rev. A, vol. 78, p. 052504, 2008.

[28] E. J. Angstmann, V. A. Dzuba, and V. V. Flambaum, "Frequency shift of hyperfine transitions due to blackbody radiation," Phys. Rev. A, vol. 74, p. 023405, 2006.

[29] M. S. Safronova and W. R. Johnson, "All-order methods for relativistic atomic structure calculations," Adv. At. Mol., Opt. Phys., vol. 55, p. 191, 2007.

[30] G. E. Brown and D. G. Ravenhall, "On the interaction of two electrons," Proc. Roy. Soc. A, vol. 208, p. 552, 1951.

[31] J. R. Mowat, "Stark effect in alkali-metal ground-state hyperfine structure," Phys. Rev. A, vol. 5, p. 1059, 1972.

[32] A. Godone, D. Calonico, F. Levi, S. Micalizio, and C. Calosso, "Starkshift measurement of the ${ }^{2} S_{1 / 2}, F=3 \rightarrow F=4$ hyperfine transition of ${ }^{133}$ Cs," Phys. Rev. A, vol. 71, p. 063401, 2005.

[33] M. S. Safronova, W. R. Johnson, and A. Derevianko, "Relativistic manybody calculations of energy levels, hyperfine constants, electric-dipole matrix elements, and static polarizabilities for alkali-metal atoms," Phys. Rev. A, vol. 60, p. 4476, 1999.
[34] C. E. Moore, "Atomic energy levels," vol. II, NSRDS-NBS 35 (U.S. Govt. Printing Office, 1971).

[35] Handbook of Basic Atomic Spectroscopic Data, http://physics.nist.gov/PhysRefData/Handbook/index.html

[36] W. R. Johnson, "Susceptibilities and shielding factors for closed-shell ions," At. Data Nucl. Data Tables, vol. 28, p. 334, 1983.

[37] E. H. Pinnington, R. W. Berendst, and M. Lumsdent, "Studies of laserinduced fluorescence in fast beams of $\mathrm{Sr}^{+}$and $\mathrm{Ba}^{+}$ions," J. Phys. B vol. 28 , p. 2095,1995

[38] J. Jin and D. A. Church, "Precision lifetimes for the $\mathrm{Ca}^{+} 4 p^{2} P$ levels: Experiment challenges theory at the $1 \%$ level," Phys. Rev. Lett., vol. 70 , p. 3213, 1993

[39] B. Arora, M. S. Safronova, and C. W. Clark, "Determination of electricdipole matrix elements in $\mathrm{K}$ and $\mathrm{Rb}$ from stark shift measurements," Phys. Rev. A, vol. 76, p. 052516, 2007.

[40] M. Gunawardena, D. Elliott, M. Safronova, and U. Safronova, "Determination of the static polarizability of the $8 s^{2} S_{1 / 2}$ state of atomic cesium," Phys. Rev. A, vol. 75, p. 022507, 2007.

[41] M. S. Safronova and C. W. Clark, "Inconsistencies between lifetime and polarizability measurements in Cs," Phys. Rev. A, vol. 69, p. 040501, 2004.

[42] J. A. Sherman, A. Andalkar, W. Nagourney, and E. N. Fortson, "Measurement of light shifts at two off-resonant wavelengths in a single trapped $\mathrm{Ba}^{+}$ion and the determination of atomic dipole matrix elements," Phys. Rev. A, vol. 78, p. 052514, 2008

[43] H. Hachisu, K. Miyagishi, S. G. Porsev, A. Derevianko, V. D. Ovsiannikov, V. G. Pal'chikov, M. Takamoto, and H. Katori, "Trapping of neutral mercury atoms and prospects for optical lattice clocks," Phys. Rev. Lett., vol. 100, p. 053001, 2008.

[44] V. A. Dzuba, V. V. Flambaum, and M. G. Kozlov, "Combination of the many-body perturbation theory with the configuration-interaction method," Phys. Rev. A, vol. 54, p. 3948, 1996.

[45] S. G. Porsev, A. D. Ludlow, M. M. Boyd, and J. Ye, "Determination of Sr properties for a high-accuracy optical clock," Phys. Rev. A, vol. 78, p. 032508, 2008.

[46] V. A. Dzuba and J. S. Ginges, "Calculation of energy levels and lifetimes of low-lying states of barium and radium," Phys. Rev. A, vol. 73, p. 032503, 2006.

[47] J. Mitroy, J. Y. Zhang, M. W. J. Bromley, and K. G. Rollin, "Blackbody radiation shift of the $\mathrm{Al}^{+}$clock transition," Eur. Phys. J. D, vol. 53, p. 15,2009

[48] S. Topcu, J. Nasser, L. M. L. Daku, and S. Fritzsche, "Ab initio calculations of external-field shifts of the 661-nm quadrupolar clock transition in neutral Ag atoms," Phys. Rev. A, vol. 73, p. 042503, 2006.

[49] K. Hosaka, S. A. Webster, A. Stannard, B. R. Walton, H. S. Margolis, and P. Gill, "Frequency measurement of the ${ }^{2} S_{1 / 2}-{ }^{2} F_{7 / 2}$ electric octupole transition in a single ${ }^{171} \mathrm{Yb}^{+}$ion," Phys. Rev. A, vol. 79, p. 033403, 2009.

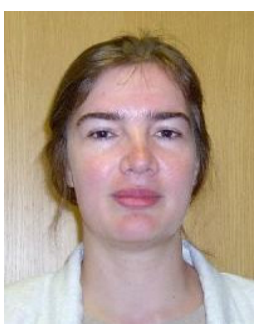

Marianna Safronova is an Associate Professor at the Department of Physics and Astronomy at the University of Delaware, working in the field of Theoretical Atomic Physics. She has received her Ph. D. from the Physics Department of the University of Notre Dame in 2001. Her Theoretical Atomic physics group focuses on the development of methodologies for high-precision atomic calculations and their applications. Her research involves both the study of the fundamental physics problems (fundamental symmetries) and applications of atomic physics to future technological developments (such as quantum computing and optical atomic clocks). 


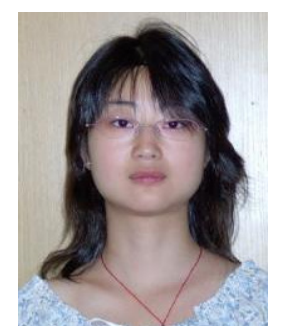

Dansha Jiang is currently pursuing a Ph. D. degree in physics at the Department of Physics and Astronomy, University of Delaware, where she is working on the development of high-precision methods for atomic calculations and their applications. Her thesis advisor is Prof. Marianna Safronova.

Bindiya Arora has received her Ph.D. from the Department of Physics and Astronomy, University of Delaware in 2008 for work on modeling of atomic systems for quantum information and atomic clocks. Her thesis advisor was Prof. Marianna Safronova.

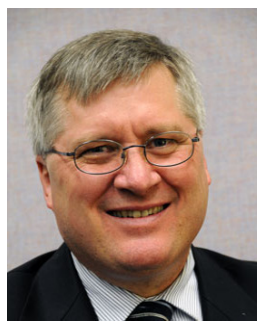

Charles W. Clark is the Chief of the Electron and Optical Physics Division of the National Institute of Standards and Technology and a Fellow of the Joint Quantum Institute, University of Maryland. He received his $\mathrm{Ph}$. D. in physics from the University of Chicago in 1979. He is also an Adjunct Professor at the Institute for Physical Science and Technology (UMCP) and a Program Manager for Atomic and Molecular Physics at the Office of Naval Research. His research interests include dynamics of ultracold atoms, physical implementation of quantum information processing, absolute optical radiometry with synchrotron radiation sources, quantitative tomographic imaging, and atomic electronic excitation in nuclear reactions.

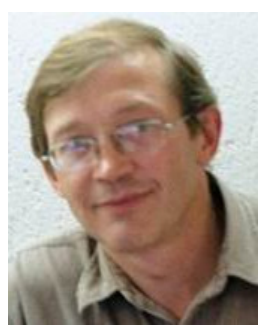

Mikhail G. Kozlov is a Leading scientific collaborator at the Molecular beam laboratory, Neutron Research Division, Petersburg Nuclear Physics Institute, Russia. He is also a member of the Foundational Questions Institute (FQXi). Dr. Kozlov received his Ph. D. from the Department of Physics of the Leningrad State University, Russia, in 1982. His research interests include development of new methods in atomic and molecular theory, parity nonconservation, time-reversal violation, and permanent electric dipole moments of atoms and molecules, space-time variation of fundamental constants, and quantum chaos.

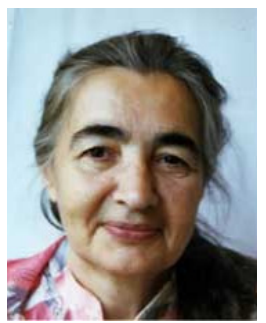

Ulyana Safronova is a Research Professor at the Department of Physics, University of Nevada, Reno, and Adjunct Professor of Physics at the University of Notre Dame. She has received Ph.D. in Physics from Vilnus University, USSR, in 1964. In 1985, she has become a Professor of Theoretical Physics, Principal Scientific Researcher at the Institute of Spectroscopy of the Russian Academy of Science, Russia. Professor Safronova's current research interests include high-precision electronic structure calculations such as relativistic many-body calculations of one, two and three valence electron systems with arbitrary core, theoretical calculation of intensity of dielectronic satellites lines, and comparison of the synthetic and experimental spectra.

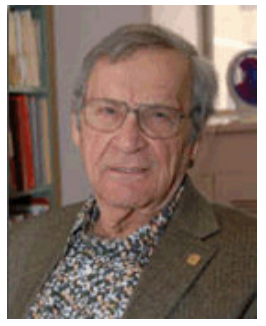

Walter R. Johnson is Frank M. Freimann Professor of Physics (Emeritus) at the University of Notre Dame. He received his $\mathrm{Ph}$. D. from the University of Michigan in 1957. Professor Johnson investigates relativistic and correlation effects in atoms using relativistic many-body methods derived from Quantum Electrodynamics. Particular attention is given to heavy atoms used in experimental studies of fundamental symmetry $(\mathrm{P})$ and $(\mathrm{T})$ violation. His current research interests also include plasma physics: average atom model and the Kubo-Greenwood theory applied to study the optical response in a warm dense plasma. 